\title{
Molecular Characterization and Expression Analysis of Pathogenesis related Protein 6 from Panax ginseng ${ }^{1}$
}

\author{
D. Myagmarjav ${ }^{a, 2}$, J. Sukweenadhi ${ }^{a, 2}$, Y. J. Kim ${ }^{b}, *$, M. G. Jang ${ }^{b}$, S. Rahimi ${ }^{a}, J_{\text {. Silva }}$, \\ J. Y. Choi ${ }^{b}$, P. Mohanan ${ }^{a}$, W. S. Kwon ${ }^{b}$, C. G. Kim ${ }^{b}$, and D.-C. Yang ${ }^{a}, \boldsymbol{b}, * *$ \\ ${ }^{a}$ Graduate School of Biotechnology and Ginseng Bank, College of Life Science, Kyung Hee University, Yongin, 446-701 Korea \\ ${ }^{b}$ Department of Oriental Medicinal Biotechnology, College of Life Science, Kyung Hee University, Yongin, 446-701 Korea \\ *e-mail: yujinkim@khu.ac.kr \\ **e-mail: dcyang@khu.ac.kr \\ Received December 26, 2016
}

\begin{abstract}
Panax ginseng Meyer is one of the important medicinal plants in the world, particularly in Asian countries. Ginseng encounters many stress exposure during its long cultivation period. However, the molecular mechanism of stress resistance is still poorly understood in spite of its importance. In this study, pathogenesis-related protein 6 (PR6), also called proteinase inhibitor (PI), was isolated from ginseng embryogenic callus, named PgPR6. The small size of PR6, containing an open reading frame of 219 bp encoding 72 amino acids, the typical characteristic of PR6 protein, shares the highest sequence similarity to PR6 of Theobroma cacao (69\% identity). Sequence and structural analysis indicated that PgPR6 belongs to class Kunitz-type PI family. This is the first report pertaining to the identification of $P R 6$ gene from the $P$. ginseng genome. The high-level expression of PgPR6 was observed in root as revealed by quantitative real-time PCR. The temporal expression analysis demonstrated that PgPR6 expression was highly up-regulated by signaling molecules, heavy metals, mechanical wounding, chilling, salt, sucrose, and mannitol stress, indicating that $P g P R 6$ may play an important role in the molecular defense response of ginseng to a various range of environmental stresses.
\end{abstract}

Keywords: Panax ginseng, abiotic stress, biotic stress, pathogenesis-related protein 6, proteinase inhibitor protein, gene expression

DOI: $10.1134 / \mathrm{S} 1022795417110060$

\section{INTRODUCTION}

Plants are continuously threatened by biotic or abiotic stresses during its growth and development. Therefore, they developed unique defense mechanisms by rapid changes in gene expression, resulting in the synthesis of specific proteins to protect them from various stresses such as pathogen attack, insect, herbivores and also other harsh growing conditions. Most inducible plant proteins under these circumstances are pathogenesis-related (PR) proteins, which are induced and accumulated in host plants as a result of biotic and abiotic stresses [1]. From the first isolation of PR protein in leaves of Nicotiana tabacum after tobacco mosaic virus infection, many proteins with similar properties have been isolated from various plants [2]. PR proteins can be divided into 17 groups (PR-1 to PR-17) on the basis of their functions, sequence characteristics, immunological relationships, enzymatic activities or other biological prop-

\footnotetext{
${ }^{1}$ The article is published in the original.

2 These authors contributed equally to this work.
}

erties and numbered in the order in which they belong [3].

Among the diverse PR families, the PR6 family is considered as the proteinase inhibitors (PIs) [4]. Plant PIs are generally small proteins with a molecular mass of 8-20 kDa, and inhibit proteolytic enzymes of animal and fungal origin, which are widespread in both monocots and dicots $[4,5]$. Among four major subclasses including serine, cysteine, aspartate and metallo-PIs based on their amino acid in the active site of the proteinases [6], the serine PIs were well characterized in various dicot species, especially in potato and tomato [5]. Serine PIs are the most abundant PR6 which is sub-divided further into Kunitz Type PI (KTI) and Bowmin-Birk PI (BBI), according to their molecular mass, location of disulfide bridge and cysteine content $[6,7]$.

The exact physiological role of PR6 according to their classes has not yet been well understood. PR6 implicates in defense against the herbivores, insects, and nematode or mechanical wounding, via interaction with the proteinases in all plant organs [6-8]. 
Even though most of PR6 studies have been focused on its role in plant-insect interactions, it has been suggested that it plays many roles in the plant response to abiotic stresses such as salt, heavy metal, water-deficit and mechanical wounding [9-12]. The identification and characterization of $P R 6$ have been demonstrated particularly in Gramineae [5], Leguminosae [13], Solanaceae families [14] and they were primarily found as a result of nutritional interest in inhibitors of animal digestive enzymes in important agricultural crops. However, most of the studies have been carried out on crop plants (cereals, legumes and solanaceous species) due to their economic importance, thus, noncrop species being comparatively neglected [15]. Korean ginseng (Panax ginseng Meyer) has been used for thousands of years as one of the most important sources of medicinal herb in Asia and is cultivated for its highly valued root used for medicinal purposes. $P$. ginseng requires 4 to 6 years cultivation period to provide the high-quality roots [16] and the root diseases are the major threat to the quality of its roots. The consecutive planting of ginseng in the same field may cause serious root diseases [17]. Therefore, to effectively manage ginseng diseases and other disorders, it is important to study and identify functional genes related to ginseng defense mechanism. Previously, we identified and characterized PR4 [16], PR10 [18] family in $P$. ginseng. In the present study, we report the cloning of PR6 gene from P. ginseng and provide the analysis on the expression profile of this gene in the defense against various environmental stresses.

\section{MATERIALS AND METHODS}

\section{Isolation of PgPR6 and Sequence Analysis}

PgPR6 ESTs were searched against the GenBank database using BLASTX algorithm. Total RNA isolated from the embryogenic callus of ginseng was previously constructed into the cDNA library [19]. Sizeselected cDNA was ligated into a $\lambda$ TriplEx2 vector and pTriplEx phagemids were excised from the $\lambda$ pTriplEx2 and used as templates for sequence analysis. The $5^{\prime}$ ends of the cDNA inserts were sequenced by an automatic DNA sequencer (ABI Prism 3700, PerkinElmer, USA). Homologous sequences of PR6 expressed sequence tag (EST) were searched against the GenBank databases using a BLASTX algorithm. A pTriplEx phagemid for $P R 6$ cDNA was excised from the $\lambda$ pTriplEx2 and used as a template for sequence analysis. Nucleotide and amino acid sequence analyses were performed using DNASIS program (Hitachi). Secondary structure analysis for PR6 homologous from plants was conducted by SOPMA [20].

These deduced amino acid sequences were utilized to search for homologous proteins via BLAST network services at the NCBI (http://blast.ncbi.nlm.nih.gov/ Blast.cgi). ClustalX [21] with default gap penalties were used to perform multiple alignments of PR6 gene sequence isolated in ginseng with other previously reported sequences from different species. A phylogenetic tree was constructed by the neighbor-joining method, and the reliability of each node was established by bootstrap methods using MEGA4 [22] software. The protein properties were estimated using ProtParam [23] and the hydropathy value, identification of conserved motifs within PR6 was accomplished with MEME [24] and a three-dimensional (3-D) model was prepared using SWISS-MODEL [25] Workspace and UCSF Chimera package, as described previously [16].

\section{Plant Materials and Application of Environmental Stresses}

P. ginseng cv. Hwang-Sook seeds (provided by Ginseng Bank) were used and cultured three-week-old plantlets were used for the treatments and RNA was extracted, as described previously [16]. For chemical stress or plant hormone treatments, the plantlets were placed for various time periods in Murashige and Skoog (MS) medium containing the indicated concentrations of chemicals: $100 \mathrm{mM} \mathrm{NaCl}, 10 \mathrm{mM}$ $\mathrm{H}_{2} \mathrm{O}_{2}, 0.2 \mathrm{mM}$ jasmonic acid (JA), $0.1 \mathrm{mM}$ abscisic acid (ABA) [16], $1 \mathrm{mM}$ salicylic acid (SA) [18], $500 \mu \mathrm{M}$ $\mathrm{CuSO}_{4}, 500 \mu \mathrm{M} \mathrm{CdSO}_{4}, 11 \%$ mannitol, $20 \%$ sucrose [39]. Chilling stress was applied by exposing the plantlets to $4^{\circ} \mathrm{C}$ [16]. For the UV treatment, the plantlets were irradiated under UVC lamps at $1.35 \mu \mathrm{E} \mathrm{m}^{-2} \mathrm{~s}^{-1}$ (below $280 \mathrm{~nm}$ ). To monitor the effect of light stress, plants were kept in the white light with an intensity of about $500 \mu \mathrm{E} \mathrm{m}^{-2} \mathrm{~s}^{-1}$ [18]. Anoxic conditions were generated by submerging whole potted plants for the duration of the experiment. For mechanical wounding stress, healthy leaves and stems of plantlets were wounded with a sterile scalpel [16]. In all cases, stress treatments were carried out in the MS media and ten plantlets were treated with each stress for $1,4,8,24$, 48 , or 72 hours. Control plants were held in a growth room at $25^{\circ} \mathrm{C}$ under a $16 \mathrm{~h}$ photoperiod.

\section{Real-Time Quantitative RT-PCR}

Total RNA was extracted from seedlings of $P$. ginseng using the RNeasy mini kit (Qiagen, Valencia, CA, USA). For RT-PCR, 200 ng of total RNA was used as a template for reverse transcription using oligo $(\mathrm{dT})_{15}$ primer $(0.2 \mathrm{mM})$ and AMV reverse transcriptase $(10 \mathrm{U} / \mu \mathrm{L})$ (INTRON Biotechnology, Inc., South Korea) according to the manufacturer's instructions. Real-time quantitative PCR was performed using $100 \mathrm{ng}$ of cDNA in a $10-\mu \mathrm{L}$ reaction volume using $\mathrm{SYBR}^{\circledR}$ Green Sensimix Plus Master Mix (Quantace, Watford, England). Specific primer for PgPR6 (5'-CCC TGG AGC AAA AAG GAG TT-3' and 5'-ACC CAA ATC CGA ACC CTA TC-3') was used to perform real-time PCR. The thermal cycler conditions recommended by the manufacturer were used as follows: 
(a)

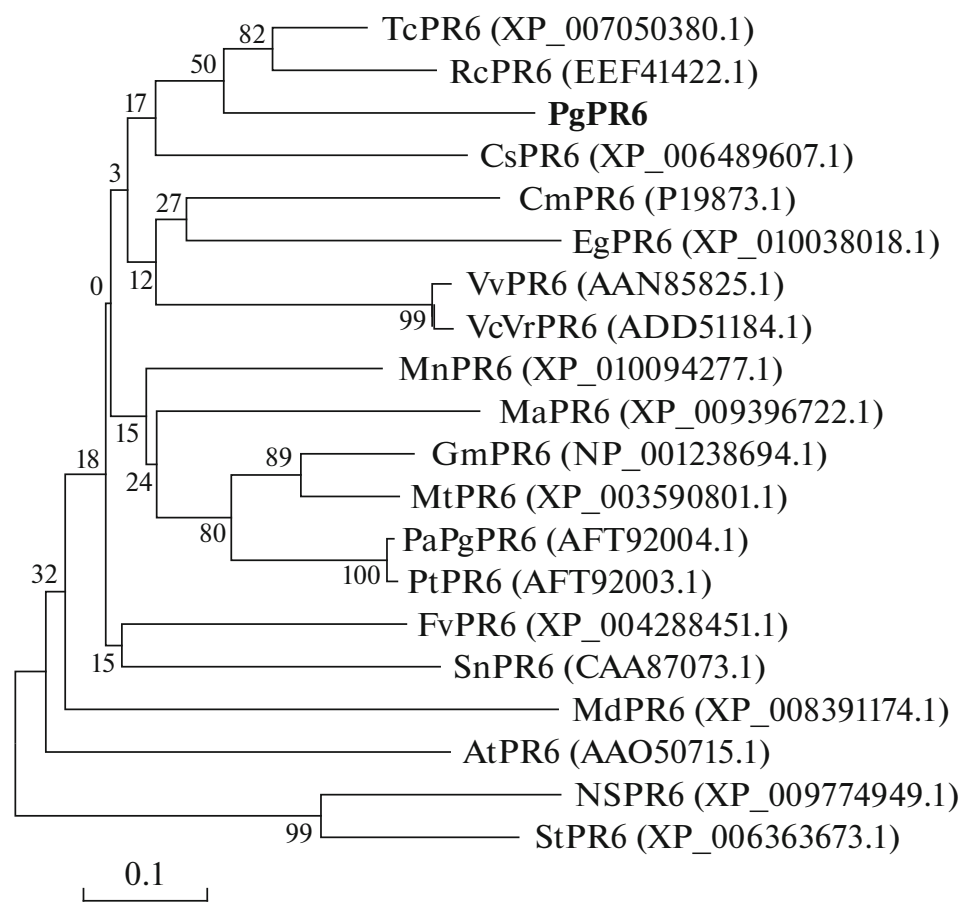

(b)

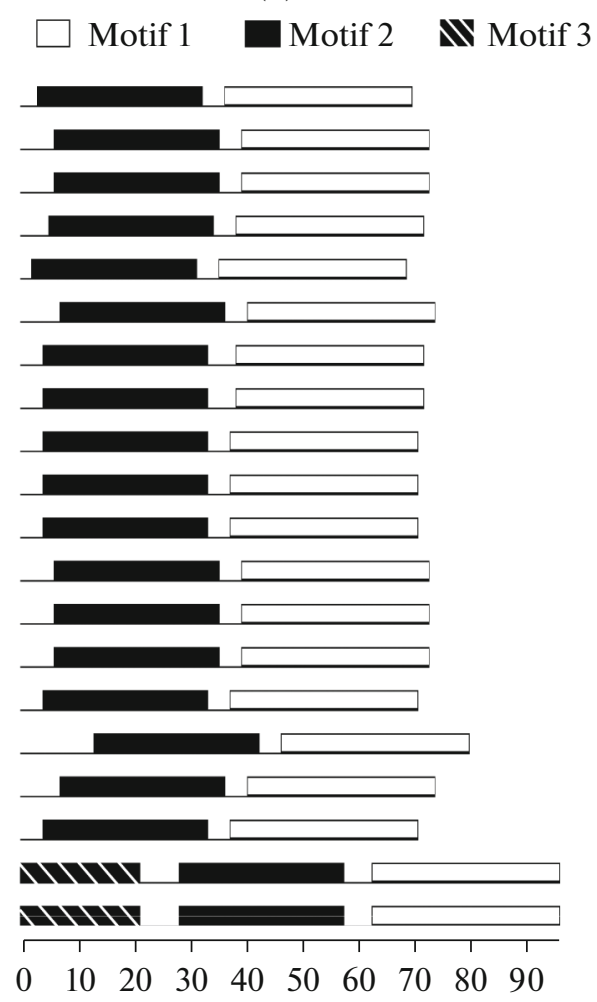

Fig. 1. Sequence homology analysis of PgPR6 with other PR6s. (a) A phylogenetic tree of PgPR6 with PR6 isozymes from various organisms including plants shown in Fig. 1. The neighbor-joining method was used, and the branch lengths are proportional to the divergence, with the scale of 0.1 representing $10 \%$ changes. (b) Organization of putative motifs in PR6 identified by MEME. Numbered color boxes represent different putative motifs. Motifs 1, 2, and 3 are indicated by the white (first), black (middle), and diagonal lines (last) boxes, respectively. Motif sizes are indicated at the bottom of the figure.

$10 \mathrm{~min}$ at $95^{\circ} \mathrm{C}$, followed 40 cycles of $95^{\circ} \mathrm{C}$ for $10 \mathrm{~s}$, $60^{\circ} \mathrm{C}$ for $10 \mathrm{~s}$, and $72^{\circ} \mathrm{C} 20 \mathrm{~s}$. Amplification, detection, and data analysis were carried out with a Rotor-Gene 6000 real-time rotary analyzer (Corbett Life Science, Sydney, Australia). The threshold cycle (Ct) represents the number of cycles at which the fluorescence intensity was significantly higher than the background fluorescence at the initial exponential phase of PCR amplification. To determine the relative fold differences in template abundance for each sample, the $\mathrm{Ct}$ value for $P g P R 6$ was normalized to the $\mathrm{Ct}$ value for $P g$ Actin and calculated relative to a calibrator using the formula $2^{-\Delta \Delta \mathrm{Ct}}$. The actin gene was selected as a reference gene [40] and its primers were designed according to the conserved region of PgActin mRNA sequences (GenBank accession no. KF699319). Three independent experiments were performed.

\section{RESULTS}

\section{Isolation and Amino Acid Sequence Analysis of PgPR6}

From the EST analysis of a cDNA library [19], we identified a cDNA clone encoding a PR6 gene. We named this gene as PgPR6 ( $P$. ginseng pathogenesisrelated protein 6); the sequence data of PgPR6 have been deposited in GenBank under accession number KP893146. The full-length cDNA of PgPR6 was 725 nucleotides long and had the putative open reading frame of $219 \mathrm{bp}$. This ORF encodes PR6 protein of 72 amino acids, beginning at the start codon ATG (position 70) and terminating at the stop codon TAA (position 288) of the cDNA.The predicted molecular weight is $17.33 \mathrm{kDa}$ and its isoelectric point is 5.38 , which is the typical characteristic of KTI $[8,13]$. The instability index (II) is calculated 24.94 and the aliphatic index, defined as a positive factor for increased thermostability was computed as 25.75 using ProtParam [23].

\section{Homology Analysis}

A GenBank BlastX search revealed that the amino acid sequence of PgPR6 has a high degree of sequence homology with PR6 from other plants. PgPR6 shows the highest sequence identity of KTI-type PR6 from Theobroma cacao (TcPR6, XP_007050380.1, 69\% identity), followed by Ricinus communis (RcPR6, EEF41422.1, 65\% identity), and Cucurbita maxima 

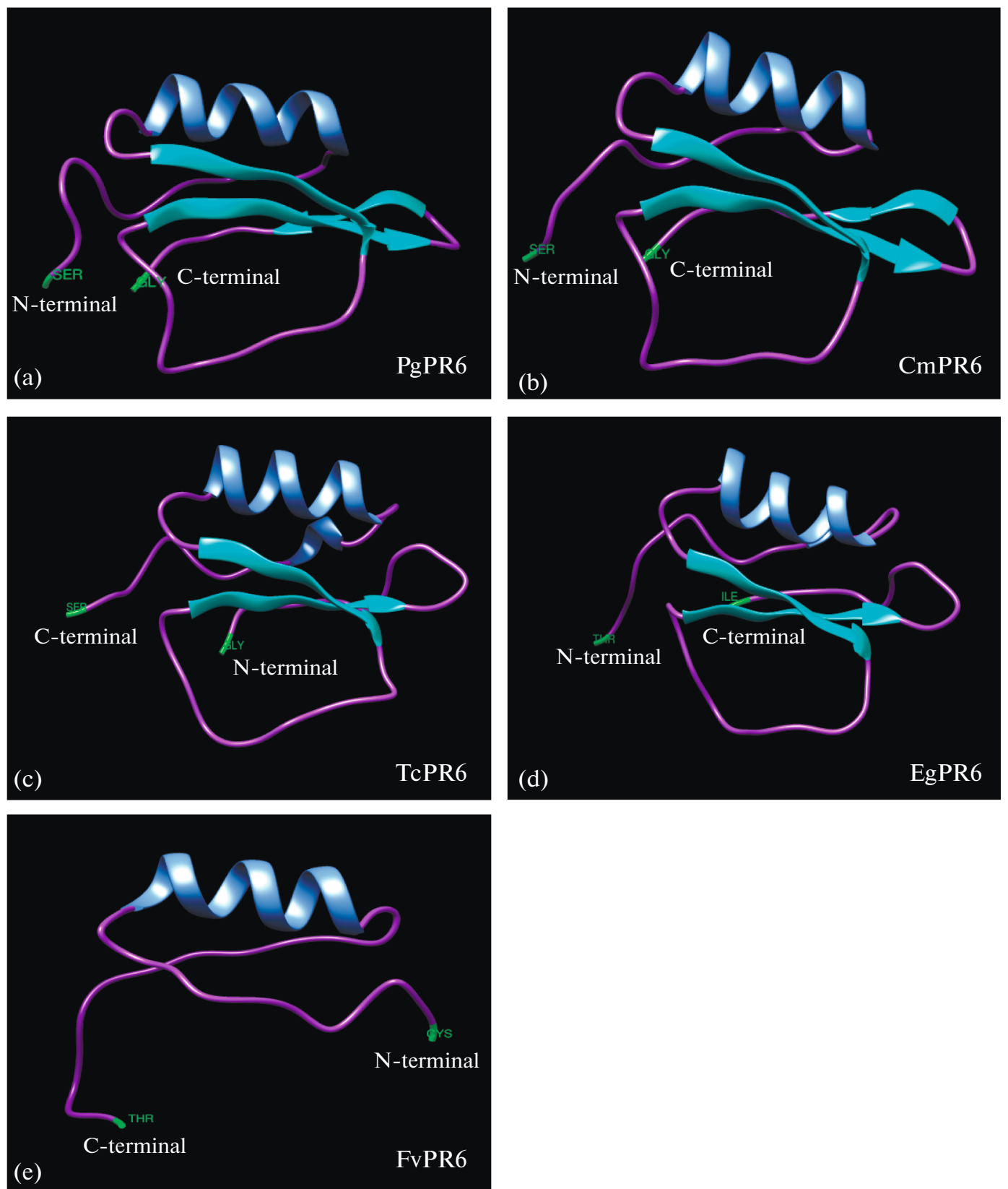

Fig. 2. The comparison of a predicted three-dimensional (3d) model of PR6. (a) P. ginseng PR6 (PgPR6), (b) C. maxima (CmPR6), (c) T. cacao (TcPR6), (d) E. grandis (EgPR6).

(CmPR6, P19873.1, 60\% identity). The protein sequence of PgPR6 and the similar proteins from other plants were used for doing multiple sequence alignment (Sup. Fig. S1) and phylogenetic tree construction (Fig. 1a). Multiple sequence alignment revealed that all amino sequences are well conserved among the known PR6 (Sup. Fig. S1). However, the $\mathrm{N}$-terminal region of PR6 protein sequences showed variance between species, except conserved Cys residue, which probably forms disulfide bridge with Cys in C-terminal. Consistently, two motifs were conserved well in all PR6s except N-terminal-located motif 3, which was shown specific to just two PR6s (Fig. 1b).

The hydrophilic profile (Sup. Fig. S2a) and secondary structure (Sup. Fig. S2b) of the estimated PR6 proteins support their similar profiles, but also shows the species-specific difference in N-terminal regions. The secondary structure analysis showed that PgPR6 consists of $14 \alpha$-helices, $11 \beta$-turns linked by 18 extended strands and 29 random coils. The composition is highly comparable to the secondary structure of EgPR6 (Eucalyptus grandis, XP_010038018.1) that 
contains $19 \alpha$-helices, $10 \quad \beta$-turns jointed by 16 extended strands, and 28 random coils and FvPR6 (Fragaria vesca, XP_004284511.1) which contains 15 $\alpha$-helices, $12 \beta$-turns linked by 18 extended strands, and 25 random coils. However, the structure profile appears more similar to the secondary structure of CmPR6 that contains $8 \alpha$-helices, $12 \beta$-turns linked by 21 extended strands, and 27 random coils (Sup. Fig. S2b) and its tertiary structure also showed most similar construction with PgPR6, having one helix chain and three turns (Figs. 4a, 4b), rather than TcPR6, EgPR6, and FvPR6 (Figs. 2c-2e).

\section{Expression Analysis of PgPR6 Gene in Diverse Organs}

To examine the expression profile of PgPR6, the quantitative RT-PCR was carried out using the cDNA templates from three organs such as leaf, stem, and root. As shown in Fig. 3, PgPR6 was highly expressed in ginseng root and very weekly expressed in the stem.

\section{Temporal Expression of PgPR6 Gene in Response to Abiotic Stresses}

The expression pattern of PgPR6 against different stresses at different time points after treatments were analyzed by real-time PCR (Fig. 4). The PgPR6 response against abiotic stress and hormone treatment demonstrated the up-regulations by all of the treatments compared to the controls $(0 \mathrm{~h})$. High light and UV exposure increased PgPR6 mRNA level up to 200fold and 2.5-fold, respectively, at $48 \mathrm{~h}$ post-treatment. Under chilling stress, PgPR6 expression decreased rapidly but accumulated at $48 \mathrm{~h}$ (5-fold) and maintained. Similarly, salt stress also increased PgPR6 expression at $48 \mathrm{~h}$ (4.2-fold). Osmotic stresses such as sucrose and mannitol highly up-regulated PgPR6 after $48 \mathrm{~h}$ post treatment $(>100$-fold and $>200$-fold, respectively). Heavy metal stresses including copper and cadmium treatments increased the expression of $P g P R 6$ at 24 and $72 \mathrm{~h}$, respectively. ABA rapidly accumulated PgPR6 mRNA at $4 \mathrm{~h}$ and maintained its upregulation until $72 \mathrm{~h}$ of treatment. Moreover, hormone treatment more strongly accumulated $P g P R 6$ mRNA, suggesting its high response to the signal molecules. JA caused the rapid and strong up-regulation of $P g P R 6$ in all the harvesting times and peaked at $24 \mathrm{~h}$ (1700-fold), in spite of the weak response against wounding treatment. PgPR6 was also strongly up-regulated but decreased to the control level after $8 \mathrm{~h}$ of SA treatment. The expression of $P g P R 6$ against $\mathrm{H}_{2} \mathrm{O}_{2}$ was up-regulated from $8 \mathrm{~h}$ and gradually increased until $72 \mathrm{~h}$ posttreatment (3500-fold).

\section{DISCUSSION}

In this study, we firstly report the isolation and characterization of the gene encoding PR6 from

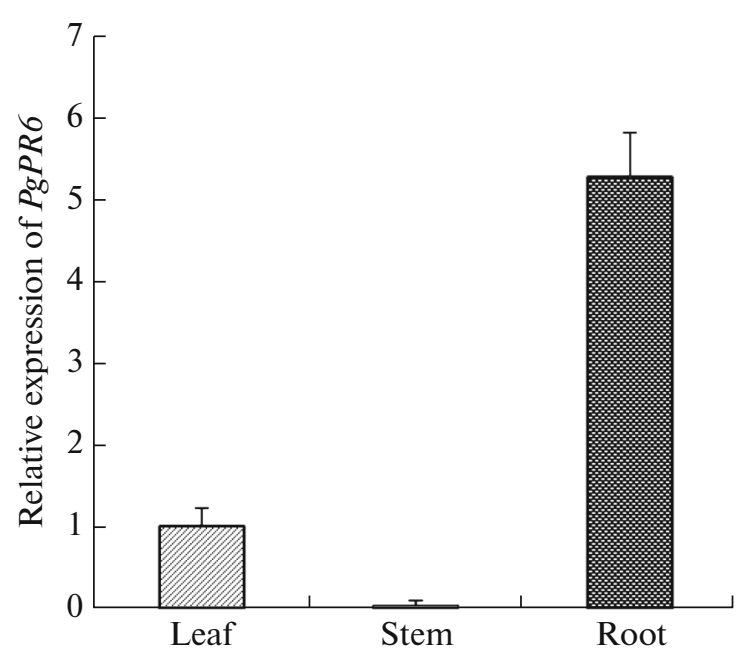

Fig. 3. Expression of PgPR6 gene in leaves, stems, and roots of Panax ginseng. Vertical bars indicate the mean value $\pm \mathrm{SE}$ from three independent experiments.

P. ginseng and its expression in response to signaling molecules, heavy metals, mechanical wounding, chilling, salt, sucrose and mannitol stresses. Sequence analysis of $P g P R 6$ showed that the full-length cDNA encoding 72 amino acids, showing typical small size of KTI-type PR6. Structural pattern of most plant KTIs are single polypeptide chain of approximately $20 \mathrm{kDa}$ with low cysteine residues (usually four), two disulfide bridges and single reactive site. The protein sequence analysis showing that PgPR6 has a single polypeptide chain of $17.35 \mathrm{kDa}$ predicted molecular mass with $2 \mathrm{Cys}$ residues which can make single disulfide bridge, led us to classify PgPR6 into KTI-type PR6. Plant KTIs are not so structurally follow the Cys-Cys pattern. For example, KTI isolated from Cajanus cajan contains five Cys residues and four of them are involved in two disulfide bridges [26], but KTI isolated from Inga laurina contains only two cysteine residues [27] which are similar to our result.

The putative amino acid sequence of PgPR6 exhibited high similarity to other PR6, particularly KTItype from other plant species, T. cacao, R. communis, and $C$. maxima containing two conserved motifs (Sup. Fig. S1). In spite of its distance on phylogenetic tree (Fig. 1a), PgPR6 and CmPR6 shares very similar tertiary structure. However, other relatives of PR6 shows different construct (Fig. 2), even though its hydropathy and secondary structures are similar (Sup. Fig. S2). This result suggests its rapid adaptive evolution of structure according to the species-dependent environment or development, which could contribute to its diversity and function of KTI [28].

The exact physiological role of PR6 has not been well reported in other species. In addition to the classical studies of PR6s related to the seed development and storage protein $[4,6]$, several reports have shown 

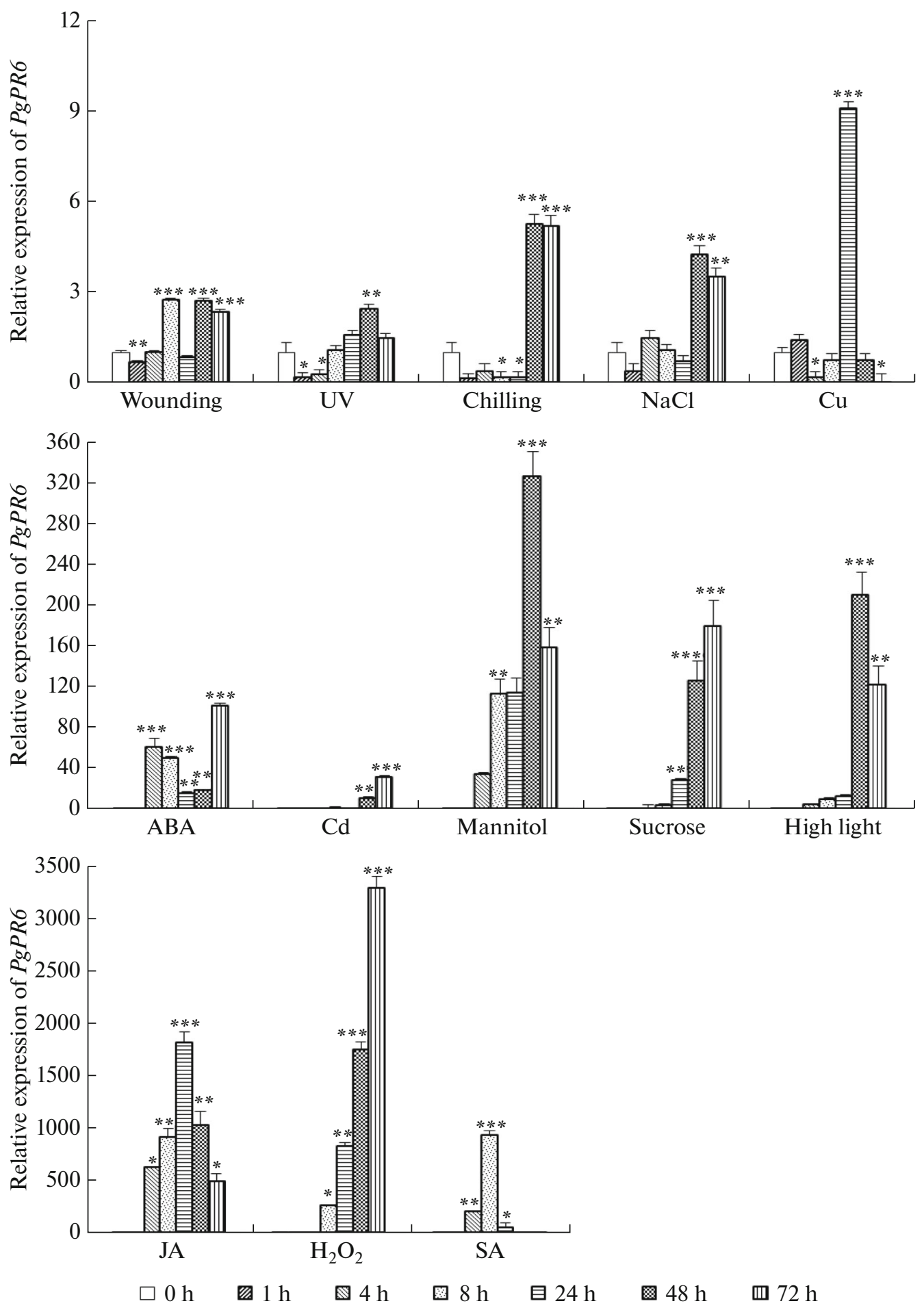

Fig. 4. Relative quantities of $P g P R 6 \mathrm{mRNA}$ at various time points (h) post-treatment with various abiotic stresses: wounding, UV light, chilling, $100 \mathrm{mM} \mathrm{NaCl}, 0.1 \mathrm{mM}$ abscisic acid, heavy metal treatment with $500 \mu \mathrm{M} \mathrm{CuSO}_{4}$, and $500 \mu \mathrm{M} \mathrm{CdSO}_{4}, 11 \%$ mannitol, $20 \%$ sucrose, high light, $0.2 \mathrm{mM}$ jasmonic acid, $10 \mathrm{mM} \mathrm{H}_{2} \mathrm{O}_{2}$, and $1 \mathrm{mM}$ salicylic acid. The error bars represent the standard error of the means of three independent replicates. Statistical significance using Student's $t$-test was assigned at $*$ for $P<$ 0.05 , ** for $P<0.01$, and ${ }^{* * *}$ for $P<0.001$, respectively, compared to non-treated plants. 
their expression in all plant organs [6,8] which may link with the general role for defense. Similar to the Populus deltoides KTI [12], the expression of PgPR6 was observed strongly in roots and weakly in leaves (Fig. 3), suggesting PgPR6 expression possibly relate to the preferred defense role in the root, as a storage protein.

The wound-induced response of PR6 has been extensively studied related with insect-resistance [29] and the up-regulation of $P g P R 6$ to wounding would be strongly mediated by hormones, such as SA, JA, and ABA [30-32]. The response to wound signaling pathway from release to transduction occurs within few minutes [33] and the high expression peak at $8 \mathrm{~h}$ and falling off after $24 \mathrm{~h}$ of PgPR6 is similar with the pattern of soybean $P R 6$ [32]. ABA acts as a primary signal in the systemic wound signaling which in turn activates the biosynthesis of JA signaling pathway, that trigger the expression of PR6 [34].

The defense response of $P g P R 6$ against heavy metals was supported by the recent reports that $P$. deltoids KTI could interact with the metal ions and up-regulated by copper stress [12], and serine PIs from Coptis japonica and Capsicum annuum contributes to the tolerance to various toxic compounds including Cd [11, 35]. The late and strong response of PgPR6 under high light exposure is consistent with BBI from Oryza sativa [5], and KTI from Amaranthus hypochondriacus [10]. Tomato PR6 was also shown to accumulate in response to UVC radiation [36] as well as sucrose [37]. Furthermore, PgPR6 expression could be positively responsive to abiotic stresses such as $\mathrm{H}_{2} \mathrm{O}_{2}$, chilling, sodium chloride, sucrose, and mannitol. The involvement of PR6 in the response of plants to abiotic stresses is connected with the enhanced proteolysis that involves during senescence of plant tissues [6].

Taken together, our results indicate that PgPR6 could play a critical role in signaling molecules, heavy metals, mechanical wounding, chilling, salt, sucrose and mannitol stress implying its involvement in the defense mechanism against biotic and abiotic stresses in ginseng. Ginseng cultivation suffers difficulties with abiotic and biotic stresses and its cultivation requires special consideration during long duration [38]. By contrast to the lack of information about ginseng genome, more $P R$ genes involved in stress have been detected and some of them identified and characterized in ginseng. Consequently, the puzzle is starting to fill although still far from complete; much more research will be needed to link them together.

\section{ACKNOWLEDGMENTS}

This research was supported by a grant from the Basic Science Research Program through the National Research Foundation (NRF), Ministry of Education, Republic of Korea to Y.J. Kim (2016R1A6A3A11931858) and Korea Institute of Planning and Evaluation for Technology in Food, Agriculture, Forestry and Fisheries IPET to D.C. Yang (112142-05-4-SB010).

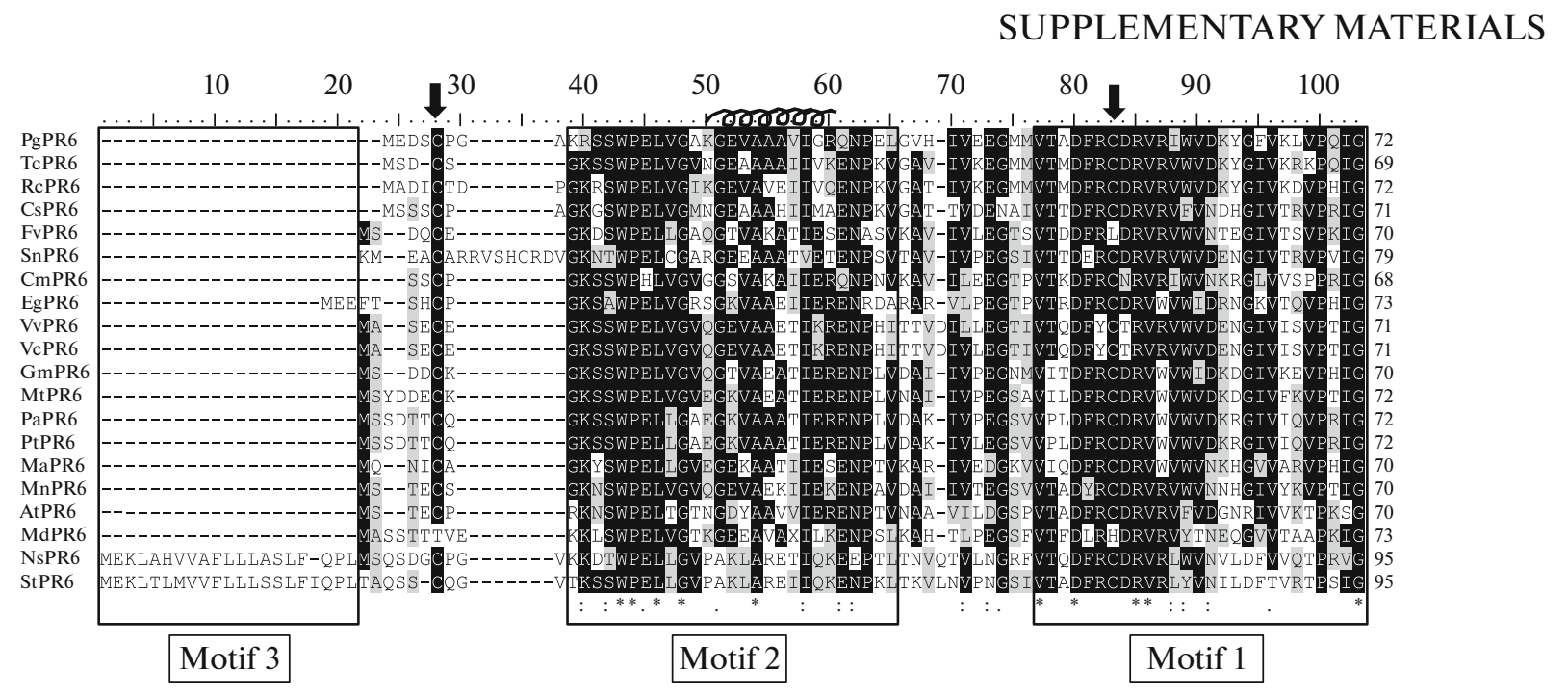

Sup. Fig. S1. Comparison of the putative amino acid sequences of PgPR6 with those of PR6s from other plants: Theobroma cacao (XP_007050380.1), Ricinus communis (EEF41422), Sambucus nigra (CAA87073.1), Vitis vinifera (AAN85825.1), Vitis cineria (ADD51184.1), Glycine max (NP_001238694.1), Medicago truncatula (XP_003590801.1), Populus alba (AFT92016.1), Populus tamentasa (AFT92015.1), Morus notabilus (XP_010094276.1), Cucurbita māxima (P19873.1,) Arabidopsis thaliana (AAO50715). A hyphen was inserted within the amino acid sequence to denote a gap. Shadowbox indicates well-conserved residues, * represents a conserved amino acid, and: represents a very similar amino acid. Arrows show the two conserved cysteines in positions 28 , 83. The conserved $\alpha$ helix domain indicated above the alignment. Three boxes show that conserved motifs obtained by MEME analysis. 


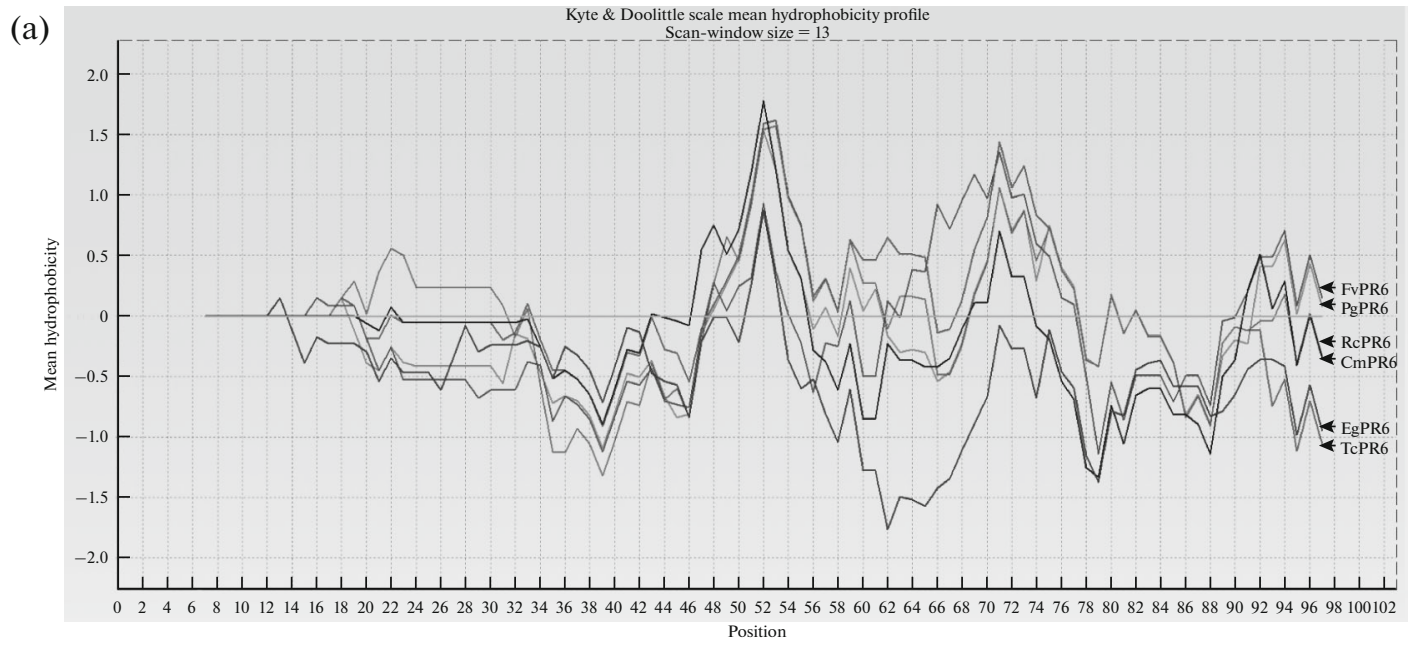

(b)
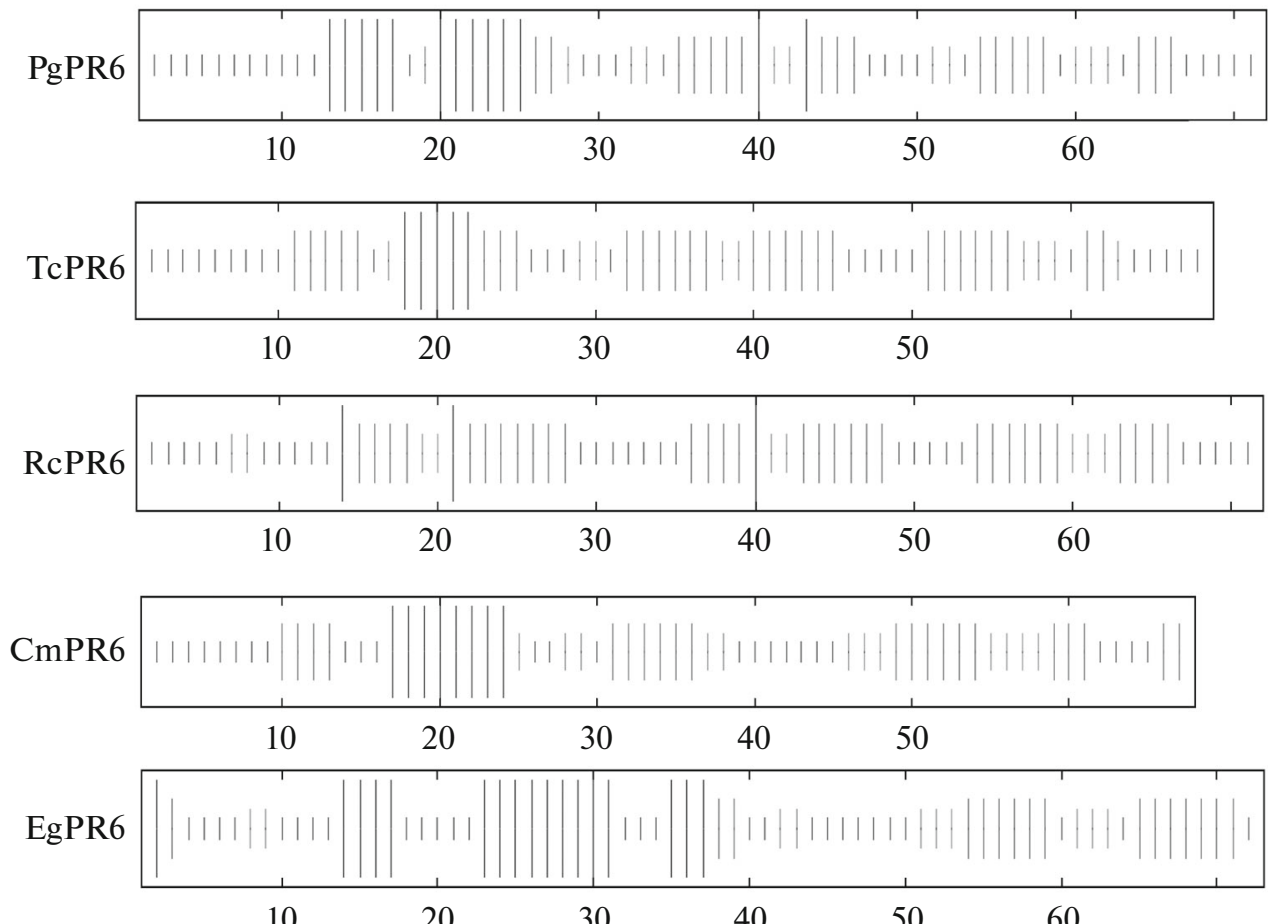

$10 \quad 20$

30

40

$50 \quad 60$

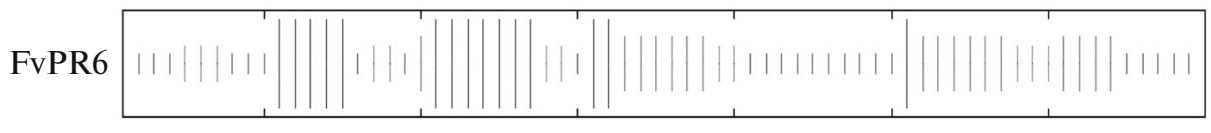

10

20

30

40

50

Sup. Fig. S2. Superimposed hydrophobicity profile of PgPR6 and secondary structure predictions for PgPR6 and homologous plant PR6s. (a) Hydrophobic domains are indicated by positive numbers; hydrophilic domains are above the line and hydrophilic domains are below the line. (b) Comparison of PR6 secondary structures by SOPMA. The helix, sheet, turn, and coil are indicated in the order from the longest to the shortest.

\section{REFERENCES}

1. Sarowar, S., Kim, Y.J., Kim, E.N., Kim, K.D., et al., Overexpression of a pepper basic pathogenesis-related protein 1 gene in tobacco plants enhances resistance to heavy metal and pathogen stresses, Plant Cell Rep., 2005, vol. 24, no. 4, pp. 216-224.
2. Niderman, T., Genetet, I., Bruyere, T., Gees, R., et al., Pathogenesis-related PR-1 proteins are antifungal (isolation and characterization of three 14-kilodalton proteins of tomato and of basic PR-1 of tobacco with inhibitory activity against Phytophtora infestans), Plant Physiol.,1995, vol. 108, no. 1, pp. 17-27. 
3. Sinha, M., Singh, R.P., Kushwaha, G.S., Iqbal, N., et al., Current overview of allergens of plant pathogenesis related protein families, Sci. World J., 2014, vol. 2014, no. 2014, pp. 1-19.

4. Sels, J., Mathys, J., Coninck B.M.A.De., Cammue, B.P.A., et al., Plant pathogenesis-related (PR) proteins: a focus on PR peptides, Plant Physiol. Biochem., 2008, vol. 46, no. 11, pp. 941-950.

5. Rakwal, R., Agrawal, G.K., and Jwa, N.S., Characterization of a rice (Oryza sativa L.) Bowman-Birk proteinase inhibitor: tightly light regulated induction in response to cut, jasmonic acid, ethylene and protein phosphatase 2A inhibitors, Gene., 2001, vol. 263, nos. 1-2, pp. 189-198.

6. Kidric, M., Kos, J., and Sabotic, J., Proteases and their endogenous inhibitors in the plant response to abiotic stress, Bot. Serbica, 2014, vol. 31, pp. 139-158.

7. Oliva, M.L.V., Silva M.C.C., Sallai, R.C., Brito, V.M., et al., A novel subclassification for Kunitz proteinase inhibitor from leguminous seeds, Biochimie, 2010, vol. 92 , no. 11 , pp. $1667-1673$.

8. Kuhar, K., Kansal, R., Mishra, A., Koundal, K.R., et al., Cloning, characterization and expression analysis of a novel gene encoding Kunitz-type protease inhibitor from Dolichos biflorus, Biotech., 2012, vol. 2, no. 3, pp. 199-209.

9. Dombrowski, J.E., Salt stress activation of woundrelated genes in tomato plants, Plant Physiol., 2003, vol. 132, no. 4, pp. 2098-2107.

10. Sanchez-Hernandez, C., Martinez-Gallardo, N., Guerrero-Rangel, A., Valdes-Rodriguez, S., et al., Trypsin and $\alpha$-amylase inhibitors are differentially induced in leaves of amaranth (Amaranthus hypochondriacus) in response to biotic and abiotic stress, Physiol. Plant., 2004, vol. 122, no. 2, pp. 254-264.

11. Joshi, R.S., Tanpure, R.S, Singh, R.K., Gupta, V.S., et al., Resistance through inhibition: ectopic expression of serine protease inhibitor offers stress tolerance via delayed senescence in yeast cell, Biochem. Biophys. Res. Commun., 2014, vol. 452, no. 3, pp. 361-368.

12. Guerra, F. P., Reyes, L., Vergara-Jaque, A., CamposHernández, C., et al., Populus deltoides Kunitz trypsin inhibitor 3 confers metal tolerance and binds copper, revealing a new defensive role against heavy metal stress, Environ. Exp. Bot., 2015, vol. 115, pp. 28-37.

13. Jamal, F., Pandey, P.K., Singh, D., and Ahmed, W., A Kunitz-type serine protease inhibitor from Butea monosperma seed and its influence on developmental physiology of Helicoverpa armigera, Process Biochem., 2014, vol. 50, no. 2015, pp. 311-316.

14. Zhang, H.Y., Xie, X.Z., Xu, Y.Z., and Wu, N.H., Isolation and functional assesment of tomato proteinase inhibitor II gene, Plant Physiol. Biochem., 2004, vol. 42, no. 5, pp. 437-444.

15. Konarev, A.V., Griffin, J., Konechnaya, G.Yu., and Shewrey, P.R., The distribution of serine proteinase inhibitors in seeds of the Asteridae, Phytochem., 2004, vol. 65, no. 22, pp. 3003-3020.

16. Kim, Y.J., Lee, H.J., Jang, M.G., Kwon, W.S., et al., Cloning and characterization of pathogenesis-related protein 4 gene from Panax ginseng, Russ. J. Plant Phyiol., 2014, vol. 61, no. 5, pp. 664-671.
17. Song, M., Yun, H.Y., Kim, Y.H., Antagonistic Bacillus species as a biological control of ginseng root rot caused by Fusarium cf. incarnate, J. Ginseng Res., 2014, vol. 38, no. 2, pp. 136-145.

18. Kim, Y.J., Jang, M.G., Lee, H.J., Jang, G.H., et al., Functional characterization of the pathogenesisrelated protein family 10 gene, $P g P R 10-4$, from Panax ginseng in response to environmental stresses, Plant Cell Tiss. Organ Cult., 2014, vol. 118, no. 3, pp. 531-543.

19. Sathiyamoorthy, S., In, J.G., Lee, B.S., Kwon, W.S., et al., In silico analysis for expressed sequence tags from embryogenic callus and flower buds of Panax ginseng C.A. Meyer, J. Ginseng Res., 2011, vol. 35, no. 1, pp. $21-30$.

20. Geourjon, C. and Deléage, G., SOPMA: significant improvements in protein secondary structure prediction by consensus prediction from multiple alignments, Comput. Appl. Biosci., 1995, vol. 11, no. 6, pp. 681-684.

21. Larkin, M.A., Blackshields, G., Brown, N.P., Chenna, R., et al., Clustal W and Clustal X version 2.0. Bioinf. Appl. Note, 2007, vol. 23, no. 21, pp. 2947-2948.

22. Tamura, K., Dudley, J., Nei, M., and Kumar, S., Mega4: molecular ecolutionary genetics analysis (MEGA) software version 4.0, Mol. Biol. Evol., 2007, vol. 24, no. 8, pp. 1596-1599.

23. Walker, J.M., The Proteomics Protocols Handbook, Totowa, NJ: Humana, 2005.

24. Atman, R., Brutlag, D., Karp, P., Lathrop, R., et al., Proceedings of the Second International Conference on Intelligent Systems for Molecular Biology, Stanford, California: AAAI, 1994, p. 28.

25. Arnold, K., Bordoli, L., Kopp, J., and Schwede, T., The SWISS-MODEL workspace: a web-based environment for protein structure homology modelling, Bioinformatics, 2006, vol. 22, no. 2, pp. 195-201.

26. Haq, S.K. and Khan, R.H., Characterization of a proteinase inhibitor from Cajanus cajan (L.), J. Protein Chem., 2003, vol. 22, no. 6, pp. 543-553.

27. Macedo, M.L.R., Carcia, V.A., Freire, M.G.M., and Richardson, M., Characterization of a Kunitz trypsin inhibitor with a single disulfide bridge from seeds of Inga laurina (SW.) Willd, Phytochem., 2007, vol. 68, no. 8 , pp. $1104-1111$.

28. Major, I.T. and Constabel, C.P., Functional analysis of the Kunitz trypsin inhibitor family in poplar reveals biochemical diversity and multiplicity in defense against herbivores, Plant Physiol., 2008, vol. 146, no. 3, pp. 888-903.

29. Oliva, M.L.V., Silva, M.C.C., Sallai, R.C., Brito, V.M., et al., A novel subclassification for Kunitz proteinase inhibitor from leguminous seeds, Biochimie, 2010, vol. 92, no. 11, pp. 1667-1673.

30. Doares, S.H., Narvaez-Vasquez, J., Conconi, A. and Ryan, CA., Salicylic acid inhibits synthesis of proteinase inhibitors in tomato leaves induced by systemin and jasmonic acid, Plant Physiol., 1995, vol. 108, no. 4, pp. $1741-1746$.

31. Rakwal, R., Agrawal, G.K., and Jwa, N.S., Characterization of a rice (Oryza sativa L.) Bowman-Birk proteinase inhibitor: tightly light regulated induction in response to cut, jasmonic acid, ethylene and protein 
phosphatase 2A inhibitors, Gene, 2001, vol. 263, nos. 1-2, pp. 189-198.

32. Graham, M.Y., Weidner, J., Wheeler, K., Pelow, M.J., et al., Induced expression of pathogenesis-related protein genes in soybean by wounding and the Phytophthora sojae cell wall glucan elicitor, Physiol. Mol. Plant Pathol., 2003, vol. 63, no. 3, pp. 141-149.

33. Leon, J., Rojo, E., and Sanchez-Serrano, J.J., Wound signaling in plants, J. Exp. Bot., 2001, vol. 52, no. 354, pp. $1-9$.

34. Dammann, C., Rojo, E., Jose, J., and Serrano, S., Abscisic acid and jasmonic acid activate wound-inducible genes in potato through separate, organ-specific signal transduction pathways, Plant J., 1997, vol. 11, no. 4, pp. 773-782.

35. Shitan, N., Horiuchi, KI., Sato, F., and Yazaki, K., Bowmin-Birk proteinase inhibitor confers heavy metal and multiple drug tolerance in yeast, Plant Cell Physiol., 2007, vol. 48, no. 1, pp. 193-197.

36. Yruela, I., Pueyo., J.J., Alonsos, P.J., and Picorel, R., Photoinhibitiona of photosystem II from higher plants effect of copper inhibition, J. Biol. Chem.,1996, vol. 271 , no. 44 , pp. $27408-27415$.

37. Johnson, R. and Ryan, C.A., Wound-inducible potato inhibitor II genes: enhancement of expression by sucrose, Plant. Mol. Biol., 1990, vol. 14, no. 4, pp. 527536.

38. Kim, Y.J., Jeon, J.N., Jang, M.G., Oh, J.Y., et al., Ginsenoside profiles and related gene expression during foliation in Panax ginseng Meyer, J. Ginseng Res., 2014, vol. 38, no. 1, pp. 66-72.

39. Purev, M., Kim, Y.J., Kim, M.K., Pulla, R.K. and Yang, D.C., Isolation of a novel catalase (Cat1) gene from Panax ginseng and analysis of the response of this gene to various stresses, Plant Physiol. Biochem., 2010, vol. 48, no. 6, pp. 451-460.

40. Liu, J., Wang, Q., Sun, M., Zhu, L., Yang, M. and Zhao, Y., Selection of reference genes for quantitative real-time PCR normalization in Panax ginseng at different stages of growth and in different organs, PLoS One, 2014, vol. 9, no. 11. e112177 